\title{
Rugosidad de tableros de fibra de densidad media (MDF) usando método de contacto y no contacto
}

\author{
Roughness of medium density fiberboards (MDF) by the contact \\ and non-contact method
}

\author{
Aldo Rolleri' ${ }^{1 *}$, Edmone Roffael ${ }^{2}$ \\ *Autor de correspondencia: ${ }^{1}$ Universidad Austral de Chile, Instituto de Tecnología de Productos Forestales, \\ Campus Isla Teja, Casilla 567, Valdivia, Chile, arolleri@uach.cl. \\ ${ }^{2}$ Georg August-Universität zu Göttingen, Institut für Holzbiologie und Holztechnologie, Deutschland.
}

\begin{abstract}
SUMMARY
The main subject of this work was to compare two methods for surface characterization; the traditional stylus or phertometer method was compared with a non-contact method (images analysis) under different climatic conditions. In this work of investigation, medium density fiberboards (MDF) were done using different raw materials and adhesives in the surface layers. In general, both techniques make a similar roughness characterization on medium density fiberboards.
\end{abstract}

Key words: roughness, moisture, MDF.

\section{RESUMEN}

El propósito de este trabajo fue comparar dos métodos de caraterización de superficies; el tradicional método de contacto (método phertometer) fue comparado con un método de no contacto (analisis de imágenes) bajo diferentes condiciones climáticas. En este trabajo de investigación, fueron fabricados tableros de fibra de densidad media (MDF) usando diferentes materias primas y adhesivos en las capas superficiales. En general, ambas técnicas hacen una caracterización similar de la rugosidad de tableros de fibras de densidad media (MDF).

Palabras clave: rugosidad, humedad, MDF.

\section{INTRODUCCIÓN}

Una de las más importantes propiedades en superficies de tableros en base a madera es la rugosidad. Esta puede definirse como la medida de finas irregularidades de la superficie. Su tamaño y frecuencia establecen la calidad de la superficie. El grado de rugosidad superficial en tableros MDF es primariamente una función de las propiedades de la madera incluyendo la especie y tamaño de las fibras. Otros factores como tipo y cantidad de resinas, ciclo de prensa, lijado y contenido de humedad de los tableros pueden también afectar las propiedades de la superficie (Hiziroglu y Graham 1998). Para entender qué significa rugosidad superficial es necesario entender qué es una superficie. Una superficie es un borde separando un objeto de otro. La superficie original llamada superficie nominal no incluye la rugosidad superficial. Cuando la superficie nominal es desviada como resultado de cualquier proceso físico o químico, la nueva superficie es llamada superficie real. Las desviaciones en la topografía de una superficie desde su estado nominal al real induce formas, ondulaciones y rugosidad. Forma es la dirección predominante de la textura de una superficie. Ondulaciones incluyen longitudes de onda que se desvían de la huella nominal. Finalmente la rugosidad considera las irregularidades más finas de una superficie, todas juntas conforman la textura de una superficie (PDI 1998).

El método común utilizado para caracterizar la rugosidad de superficies de maderas y productos base madera es el llamado método por contacto de acuerdo a la norma DIN 4768 (Sander 1989). La caracterización de una superficie a través del método de contacto emplea parámetros matemáticos y estadísticos para su interpretación. El parámetro más importante es la rugosidad media $\left(R_{a}\right)$. Como se puede ver en la figura $1, R_{a}$ está determinado como la altura de un rectángulo con un largo Lm y de la misma área que la superficie entre el perfil de la superficie y la línea media (Östman 1983).

Una de las principales desventajas de este método es el relativo largo tiempo necesario para lograr varias medidas. Sin embargo, este método es muy restrictivo por- 


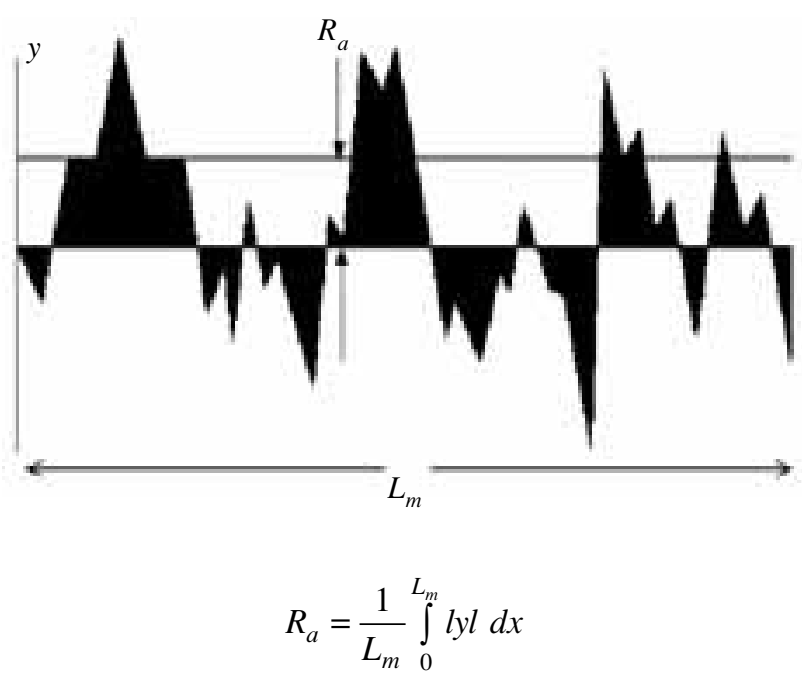

Figura 1. Rugosidad promedio $\left(R_{a}\right)$ en función de la altura de un rectángulo con un largo $L_{m}$ (Östman, 1983).

Average roughness $\left(R_{a}\right)$ in function of the height of a rectangle with length $L_{m}$ (Östman, 1983).

que una medición traza sólo una corta y pequeña línea. En las últimas décadas la investigación se ha centrado en desarrollar técnicas alternativas de no contacto más eficientes. Uno de los principios de estos métodos de no contacto es la medición de la intensidad de luz reflejada desde la superficie de la muestra y evaluar la luz reflejada por medio de sensores ópticos. Los sensores ópticos miden la rugosidad, teniendo la ventaja, respecto a los métodos de contacto de permitir altas velocidades en la adquisición de datos desde un área relativamente grande (Devoe et al. 1992). La caracterización de superficies de maderas por el método de no contacto ha sido desarrollada con el propósito de encontrar una herramienta rápida para controles de calidad compatibles con los procesos de manufacturado (Lefevre 1998).

Análisis de imágenes es una técnica emergente en el campo de la robótica. La primera función de esa técnica es capturar una imagen desde una videocámara y almacenarla en la memoria de un computador para ser procesada (Faust 1987). El análisis de imágenes toma datos de un plano bidimensional, datos que son registrados a través de una fotografía digital. Cada píxel en la imagen tiene un eje de coordenadas $\mathrm{X}-\mathrm{e} \mathrm{Y}-$. El número de píxeles en una imagen determina la resolución de la fotografía, típicamente éstas tienen $512 * 512$ píxeles. Normalmente son usados 257 niveles proporcionales de intensidad de luz; cada píxel puede tomar un valor entre 0 y 256. Cada posible valor es asociado a un nivel de grises entre negro (0) como un valor mínimo y blanco (256) como un valor máximo. Esto determina los límites de contraste y brillo. El principio se puede apreciar en la (figura 2), donde es utilizada una cámara digital. Esta cámara recolecta la luz reflejada desde una muestra. La imagen digital es procesada por un computador que examina el patrón de la luz dispersa de la imagen y calcula los parámetros de una superficie con un histograma del nivel de grises de ésta.

La rugosidad óptica es calculada como la desviación estándar del histograma de niveles grises [1]. La desviación estándar es calculada como sigue:

$$
S D=\sqrt{\frac{1}{n-1} \sum_{i=0}^{255} F_{i}\left(x_{i}-\bar{x}\right)^{2}}
$$

donde:

$\mathrm{n}=$ píxeles en la imagen, $\mathrm{xi}=$ nivel de grises $(=\leq \mathrm{i} \leq$ 256), $\mathrm{Fi}=$ frecuencia de píxeles para un nivel de grises xi.

El principal objetivo de este estudio fue comparar el método de no contacto con el de contacto en la capacidad de medir rugosidad superficial en tableros de fibras de densidad media (MDF). Fueron estudiados diferentes aspectos relacionados con las propiedades de las superficies de tableros de fibras de densidad media los que incluyen: a) efecto de fibras frescas, fibras recicladas de tableros encolados con resina UF y partículas de corcho en la superficie de tableros MDF, donde se usaron resinas melamina-urea-formaldehído (MUF) y tanino-formaldehído (TF) y b) efecto del almacenamiento bajo tres condiciones medioambientales $\left(20{ }^{\circ} \mathrm{C} / 30 \%\right.$ humedad relativa, $20{ }^{\circ} \mathrm{C} / 65 \%$ humedad relativa y $20{ }^{\circ} \mathrm{C} / 85 \%$ humedad relativa) en la rugosidad superficial de tableros de fibras de densidad media.

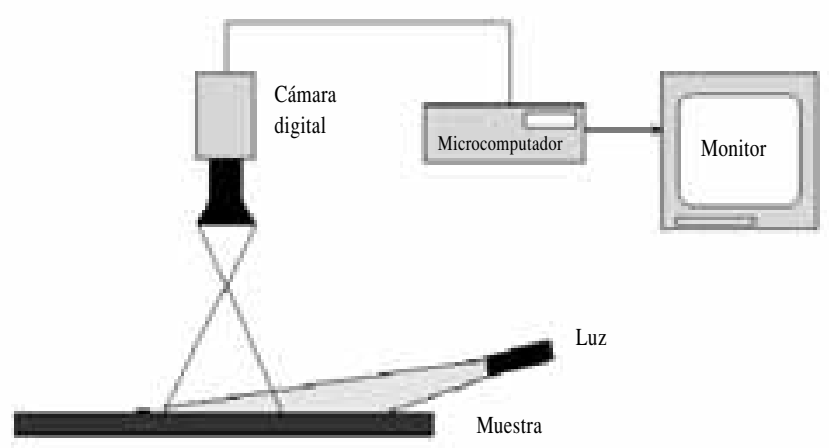

Figura 2. Configuración de dispositivos para medir rugosidad superficial por el método de no contacto.

Hardware for measuring surface roughness by the noncontact method. 


\section{MÉTODOS}

Se fabricaron tableros de fibras de densidad media (MDF) de una y tres capas usando diferentes materias primas en las capas superficiales y diferentes adhesivos. El material para la superficie fue: corcho reciclado, fibras frescas industrialmente producidas (pulpa termomecánica, PTM), fibras recicladas generadas desde tableros de fibra de densidad media, usando urea formaldehído UF como adhesivo. Las fibras frescas (PTM) fueron suministradas por una compañía alemana de tableros MDF. La pulpa fue obtenida de una mezcla de Picea abies (L.) Karst. y Pinus sylvestris L. El pulpaje se realizó a una temperatura de $180^{\circ} \mathrm{C}$. El material desfibrado fue secado en un horno a $70{ }^{\circ} \mathrm{C}$ hasta un contenido de humedad del $5 \%$.

Las fibras recicladas fueron producidas por un proceso termohidrolítico, el proceso consistió en: los tableros MDF no recubiertos, donde se utilizó urea-formaldehído UF fueron cortados en piezas de $5,0 \mathrm{~cm} * 5,0 \mathrm{~cm}$, para luego ser reducidos con una máquina golpeadora (Electra industrie). Después de esta etapa, el material con una granulometría inferior a $0,5 \mathrm{~mm}(<0,5 \mathrm{~mm})$ fue rechazado. Este material clasificado fue introducido en un autoclave de 50 litros usando $1 \%$ de hidróxido de sodio $(\mathrm{NaOH})$ (base seca) con una relación de licor 1:6. El autoclave fue calentado hasta una temperatura máxima de $130{ }^{\circ} \mathrm{C}$. Alcanzada esta temperatura la cocción se continuó por 1 hora. Durante el pulpaje el autoclave rotaba sobre un eje en $360^{\circ}$. Después de la cocción las fibras fueron enfriadas dentro del autoclave a temperatura ambiente por 18 horas. A continuación, las fibras obtenidas fueron secadas a $70{ }^{\circ} \mathrm{C}$ hasta un contenido de humedad del $5 \%$. Posteriormente las fibras fueron procesadas en un molino Pallmann-mill PXL 18 a 12.400 rpm para formar una pelusa. Las fibras fueron tamizadas, separándose las fibras finas de las gruesas. Para la producción de MDF reciclado fueron usadas sólo fibras recicladas entre $0,2 \mathrm{~mm}$ y $2,0 \mathrm{~mm}$ en las capas superficiales.

Las partículas de corcho fueron suministradas por una compañía alemana. Las partículas de corcho fueron tamizadas y clasificadas en el rango de 0,2-1 mm. Luego fueron secadas hasta aproximadamente $2,0 \%$ de contenido de humedad.

Para la preparación de los tableros de fibra de densidad media se utilizó como adhesivo una resina comercial de melamina-urea-formaldehído (MUF), BASF K 407 con un contenido de melamina de alrededor de $1 \%$ y una resina tanino-formaldehído (TF).

Se fabricaron seis diferentes series (tipos) de tableros de fibra de densidad media. De cada serie se produjeron tres tableros, completando 18 unidades. Se fabricaron tableros MDF monocapas tanto en el caso de fibras frescas como recicladas. Para el caso de tableros MDF tricapa, estos se componían de partículas de corcho recicladas en las capas superficiales y fibras frescas en la capa media.
Después de prensar y enfriar los tableros, estos fueron dimensionados hasta un tamaño de $410 \mathrm{~mm} * 410 \mathrm{~mm}$ y lijados con un grano de 120 hasta un espesor final de 13 $\mathrm{mm}$. Los 18 tableros MDF fueron acondicionados en un primer paso a un clima de $20{ }^{\circ} \mathrm{C} / 30 \%$ de humedad relativa hasta humedad de equilibrio.

Se midió la rugosidad superficial de los MDF usando el método de contacto (Perthometer (S4P)) y el método de no contacto (Análisis de Imagen, Leica Q500MC y cámara JVC-CCD). A continuación los tableros fueron acondicionados a un clima de $20^{\circ} \mathrm{C}$ y $65 \%$ de humedad relativa para aumentar la humedad hasta que los tableros alcancen el contenido de humedad de equilibrio. Los mismos parámetros fueron medidos en una última etapa de acondicionamiento a $20^{\circ} \mathrm{C}$ y $85 \%$ de humedad relativa para evaluar nuevamente la rugosidad superficial.

En este estudio se determinó el grado de interacción entre factores (variables independientes) y respuestas (variables dependientes) de los tableros de fibras sin recubrir. Los datos recogidos fueron estadísticamente analizados con análisis de varianza y prueba de multicomparación de Tukey. Los factores (variables independientes) fueron: materia prima (fibras frescas, fibras recicladas, partículas de corcho), adhesivos melamina-urea-formaldehído (resinas-MUF), tanino-formaldehído (resinas-TF), condiciones climáticas $\left(20^{\circ} \mathrm{C} / 30 \%\right.$ h.r., $20^{\circ} \mathrm{C} / 65 \%$ h.r., $20{ }^{\circ} \mathrm{C} / 85 \%$ h.r.) y las respuestas (variables dependientes) fueron: rugosidad media $R_{a}(\mu m)$, contenido de humedad (\%). Los factores o la interacción de estos provocan diferencias significativas en los valores de las respuestas (propiedades de los tableros de fibras).

Finalmente, el tradicional método de contacto fue comparado con el de no contacto (análisis de imágenes). La comparación fue hecha probando tableros de densidad media (MDF) bajo las diferentes condiciones climáticas.

\section{RESULTADOS}

A continuación se presentan los resultados indicando la influencia de la materia prima, tipo de adhesivo y condiciones climáticas en la rugosidad superficial de los tableros de fibras de densidad media MDF sin recubrir y encolados con resinas MUF- y TF a través de los métodos de contacto y no contacto.

Método de contacto. La figura 3 muestra los valores de rugosidad por contacto $R_{a}$ para toda la serie de tableros de fibra. En general, el método de contacto $R_{a}$ muestra que tableros encolados con resinas TF tienen superficies más suaves que aquellos donde se usaron resinas MUF, independiente de la materia prima empleada. Tableros de fibra MUF incrementan su rugosidad con un mayor contenido de humedad en el tablero, sin embargo, en el caso de MDF encolados con resinas TF sólo fue observado un 

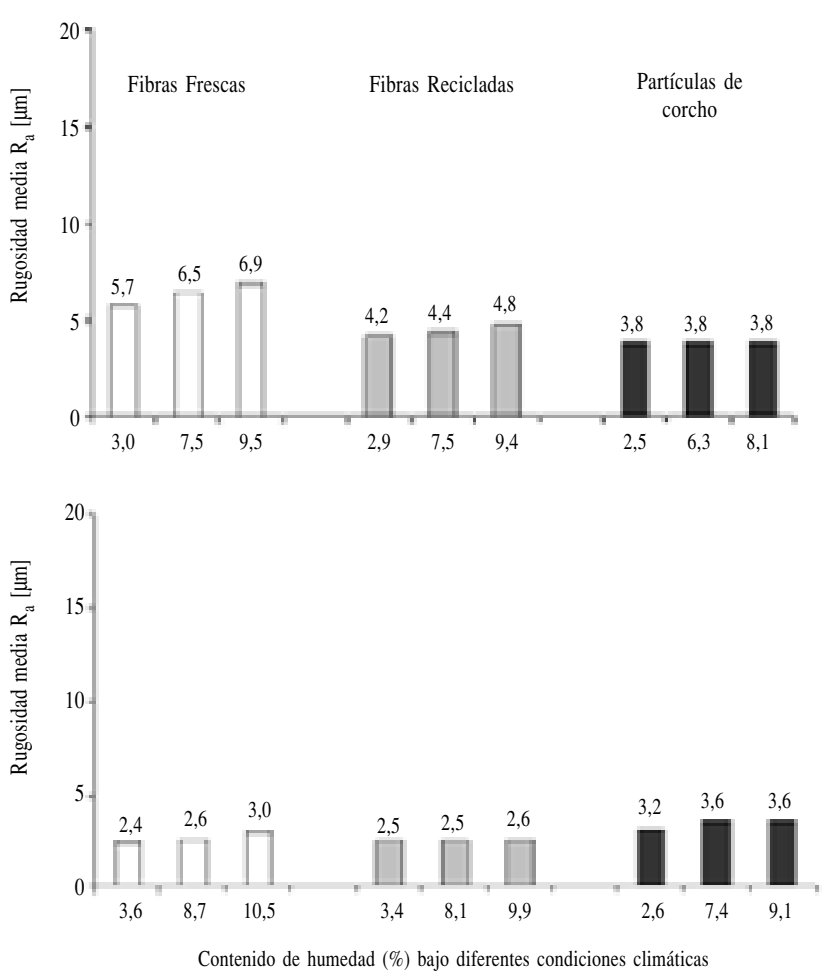

Figura 3. Rugosidad promedio $R_{a}(\mu m)$ de tableros no recubiertos (MDF) donde se usó resinas MUF (a) - y TF (b), fabricados con diferentes materias primas en las capas superficiales y tres climas $\left(20^{\circ} \mathrm{C} / 30 \%, 20{ }^{\circ} \mathrm{C} / 65 \%, 20^{\circ} \mathrm{C} / 85 \%\right)$.

Average roughness $R_{a}(\mu m)$ of uncoated MUF (a) and TF (b) - bonded medium density fiberboards (MDF), made using different raw materials in the surface layers under three climatic conditions $\left(20{ }^{\circ} \mathrm{C} / 30 \%, 20{ }^{\circ} \mathrm{C} / 65 \%, 20{ }^{\circ} \mathrm{C} / 85 \%\right)$.

leve incremento en la rugosidad, debido a un incremento en el contenido de humedad.

El análisis estadístico indica que la materia prima lignocelulósica es el factor más importante que afecta la rugosidad de tableros de fibras encolados con adhesivos MUF y TF. La prueba estadística hace presente una interacción significante entre la materia prima y los adhesivos. Esa interacción fue analizada usando la prueba de Tukey. Una comparación entre materias primas por cada adhesivo indica que la rugosidad de tableros de fibras MUF hechos con fibras frescas es significativamente diferente si se compara con la rugosidad de superficies de tableros de fibras TF hechos con fibras frescas y recicladas. La rugosidad de tableros de fibras $\mathrm{TF}$ hechos con particulas de corcho es significativamente diferente a la encontrada para los tableros de fibras TF hechos con fibras frescas y recicladas.

En lo concerniente al contenido de humedad en los tableros, las condiciones climáticas son los factores dominantes. Sin embargo, a través de la prueba de Tukey se encontró que los adhesivos y la materia prima también pueden influenciar en el contenido de humedad.
Método de no contacto. La rugosidad superficial de tableros de fibras de densidad media fue evaluada usando análisis de imágenes. La figura 4 muestra el efecto del material lignocelulósico y del contenido de humedad en la rugosidad óptica (DS) de tableros de fibras de densidad media no recubiertos encolados con resinas MUF. El resultado revela que en estos tableros el método de no contacto utilizando análisis de imágenes puede detectar el efecto del contenido de humedad en la rugosidad superficial. Independientemente de la materia prima usada, la desviación estándar (DS) incrementa cuando el contenido de humedad de los tableros incrementa. En general, tableros de fibras de densidad media no recubiertos y encolados con fibras frescas muestran los más altos valores de DS. Por otra parte, los resultados indican que los valores más bajos de rugosidad óptica (DS) fueron encontrados en tableros MDF de fibras recicladas encoladas con resinas MUF. Los valores de DS de los MDF recubiertos con partículas de corcho encoladas con resinas MUF tomaron una posición intermedia.

La figura 4 muestra además el efecto del material lignocelulósico y del contenido de humedad en la rugo-
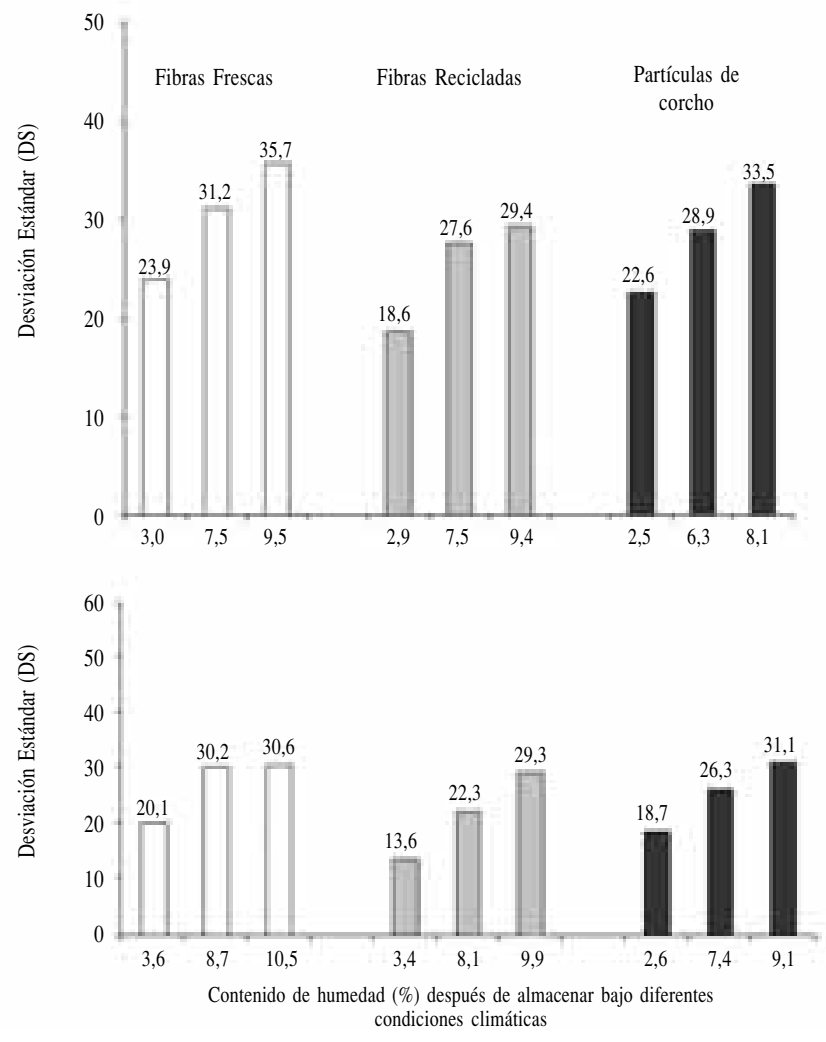

Figura 4. Desviación estándar (DS) de tableros no recubiertos MDF con resinas MUF (a) y TF (b), hechos de diferentes materias primas en las capas superficiales.

Standard deviation (SD) of uncoated MUF (a) and TF (b)-bonded medium density fiberboards MDF, made using different raw materials in the surface layers. 
sidad superficial de tableros de fibra de densidad media con superficies no recubiertas y encoladas las fibras con resinas TF. Los resultados son similares a aquellos encontrados para tableros MDF encolados con resinas MUF. Aquí también la técnica de análisis de imágenes detecta el efecto del contenido de humedad en la rugosidad superficial de tableros encolados con resinas TF. El tablero MDF manufacturado con fibras frescas encoladas con resinas TF muestra en general valores más altos de rugosidad, seguidos por MDF recubierto con partículas de corcho encoladas con resinas TF. Los valores más bajos fueron encontrados para MDF de fibras recicladas encoladas con resinas TF.

Finalmente, en la figura 5, el tradicional método de contacto es comparado con el de no contacto (análisis de imágenes). Los resultados muestran la alta sensibilidad del método de no contacto hacia cambios en las condiciones climáticas en comparación con el método de contacto, donde los valores $R_{a}$ de los tableros probados no cambian mucho debido a cambios en las condiciones climáticas. En general, sin embargo, ambas técnicas realizan una caracterización similar de la rugosidad en superficies de tableros de densidad media (MDF).

\section{DISCUSIÓN}

El contenido de humedad de equilibrio de tableros de fibras de densidad media MDF incrementa con un aumento de la humedad relativa independiente del material lignocelulósico empleado para la manufactura de los tableros. Sin embargo, la influencia de los adhesivos usados en el contenido de humedad no deja de ser relevante teniendo en cuenta que tableros MDF encolados con resinas TF poseen en general un contenido de humedad de equilibrio mayor en comparación con los tableros MDF donde se usaron resinas MUF. Esto es debido a la naturaleza alcalina de las resinas TF.

Una comparación entre las materias primas utilizadas indica que las fibras frescas producen superficies más rugosas que las recicladas. Estas últimas de una longitud menor y diámetro original del lumen disminuido, resultado de los procesos de reciclado del tablero, producen fibras más planas y flexibles que mejoran los enlaces entre ellas produciendo superficies más suaves. Por otra parte, tableros MDF donde se usaron resinas MUF y TF y que fueron recubiertos con corcho no muestran grandes cambios en la rugosidad cuando el contenido de humedad del tablero se incrementa. Esto es debido a la presencia de suberina, que es un compuesto químico presente en el corcho que disminuye su higroscopicidad.

A partir de los resultados de las figuras 3 y 4 es posible visualizar que, en general, tanto el método de contacto como el de no contacto, para tableros MDF encolados
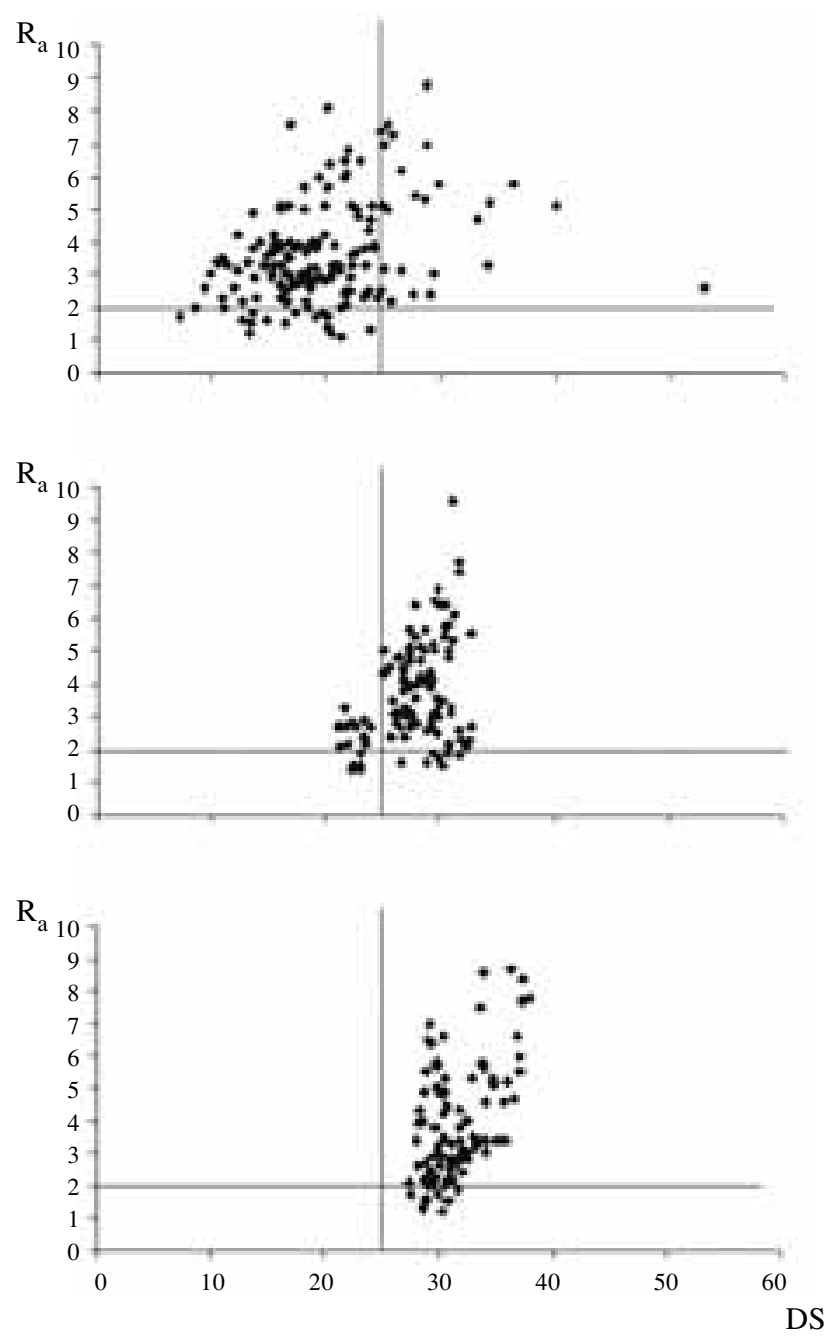

Figura 5. Efecto de las condiciones climáticas en la rugosidad promedio $\left(R_{a}\right)$ y la desviación estándar (DS) de tableros (MDF) sin recubrir encolados con resinas MUF- y TF, fabricados usando diferentes materiales lignocelulósicos. Los tableros fueron almacenados bajo diferentes condiciones climáticas $20{ }^{\circ} \mathrm{C} / 30 \% \mathrm{hr}$ (a), $20{ }^{\circ} \mathrm{C} / 65 \% \mathrm{hr}$ (b) y $20^{\circ} \mathrm{C} / 85 \% \mathrm{hr}$ (c).

Effect of climatic conditions on the average roughness $\left(R_{a}\right)$ and standard deviation (SD) of uncoated UF- and TF-bonded particleboards and MUF- and TF-bonded medium density fiberboards (MDF), made using different lignocellulosic raw materials; stored under different climatic conditions $\left(20{ }^{\circ} \mathrm{C} / 30 \%\right.$ rh (a), $20{ }^{\circ} \mathrm{C} / 65 \%$ rh (b) and $20{ }^{\circ} \mathrm{C} / 85 \%$ rh (c).

con TF, obtienen superficies más suaves respecto de tableros MDF encolados con MUF. Como se puede ver en este estudio, un aumento de la humedad de equilibrio incrementa los niveles de rugosidad de un tablero, pero en función del tipo de fibra utilizado como también el adhesivo aplicado. Tableros donde el adhesivo TF permitió un contenido de humedad de equilibrio mayor que aquellos encolados con MUF para un mismo nivel de humedad relativa, presentaron superficies más suaves debido a que el maquinado que creó las superficies, en este 
caso un proceso de lijado, fue realizado sobre fibras más plastificadas. Los resultados de los análisis estadísticos confirman que las variables dependientes (contenido de humedad de equilibrio y rugosidad media) están en función de una compleja interacción de muchas variables.

\section{CONCLUSIONES}

La rugosidad de los tableros MDF aumenta al usar adhesivos MUF y TF, en la medida que el contenido de humedad en los tableros se incrementa.

El material lignocelulósico es el factor más importante que afecta la rugosidad de tableros MDF cuando se usan adhesivos MUF y TF.

Tableros MDF fabricados con adhesivo TF, en general presentan superficies más suaves que aquellos donde se utilizaron adhesivos MUF.

El método de no contacto puede detectar cambios en la rugosidad de tableros de fibra MDF con adhesivos MUF y TF, debido a la influencia de la materia prima y las condiciones climáticas.

Ambas técnicas parecen tener diferentes sensibilidades a cambios en las condiciones climáticas.

\section{REFERENCIAS}

Devoe D, L Knox, G Zhang. 1992. An experimental study of surface roughness assessment using image processing. Technical Research Report. Mechanical Engineering Department and Systems Research Center. University of Maryland at College Park MD 20742. TR 92-28. 6 p.

Faust DT. 1987. Real measurement of veneer surface roughness by image analysis. Forest Products Journal 37(6): 34-40.

Hiziroglu S, M Graham. 1998. Effect of press closing time and target thickness on surface roughness of particleboard. Forest Products Journal 48(3): 50-54.

Lefevre R. 1998. Surface inspections on wood based panels: First results and possibilities of development. In Publication $3^{\text {rd }}$ Eurowood Symposium. 7 p.

Östman B. 1983. Surface roughness of wood based panels after aging. Forest Products Journal 33(7): 35-42.

PDI 1998. Surface metrology guide-home. Precision Devices Inc. Consultado 15 nov. 2000. Disponible en http:// www.predev.com/smg/.

Sander M. 1989. Oberflächen-Messtechnik für den Praktiker. Göttingen, Alemania. Selbstverlag der Feinprüf-Perthen GmbH. 18p. 